\title{
Arbor
}

\section{Bases científicas del entrenamiento deportivo}

\section{José Luis Martínez Rodríguez}

Arbor CLXV, 650 (Febrero 2000), 127-151 pp.

Si entendemos por entrenamiento deportivo todo proceso formativo organizado que tiene por finalidad el rápido incremento de las capacidades fisicas, psíquicas, espirituales y técnico-motrices de los individuos, jel entrenamiento existió desde el primer momento de la aparición del ser humano en la tierra!

El entrenamiento comenzó a ser sistematizado con la aparición de los primeros ejércitos organizados, es decir, como preparación militar.

Grecia, al crear las primeras grandes competiciones deportivas, dio origen a la metodología del entrenamiento deportivo, y llegó a descubrir casi todas las bases del entrenamiento deportivo, de forma más o menos empírica.

El entrenamiento deportivo científico se origina en la primera mitad del siglo XX, y se consolida en las décadas 60 y 70, gracias a las aportaciones de especialistas rusos como Ozolin, Mastveev, Vorobjev, Verchoshanskij, etc.

En la actualidad, el proceso de entrenamiento se plantea como una «cadena operativa», un paso metodológico que incluye un gran número de especialistas de ciencias auxiliares. A pesar de estos completísimos equipos de trabajo, el resultado deportivo, el éxito deportivo final, sigue dependiendo, en muy alto porcentaje, del factor humano, es decir del deportista mismo, del hombre en definitiva..

«Hay seres para quienes el deseo de saber no se sabe a sí mismo»

Salvador de Madariaga 
«En el principio era él pié. Hace cuatro millones de años, antes de adquirir el uso de la palabra o de la razón, nuestros antepasados ya caminaban erguidos sobre dos pies». Así comienza Marvin Harris su libro "Nuestra especie". Y continúa, «nuestros antepasados simios eran diferentes de los otros simios. Tenían pies como los nuestros, cuyos dedos no podían doblarse para asir o recoger objetos y que servían principalmente para permanecer de pié, correr, saltar o dar patadas. Todo lo demás era responsabilidad de las manos... Un simio bípedo y bimano solo tiene sentido desde el punto de vista de la evolución, porque podía hacer en el suelo algo que ninguna otra criatura había hecho nunca, tanto ni tan bien: utilizar las dos manos para fabricar y transportar herramientas, y utilizar herramientas para satisfacer las necesidades cotidianas».

¡Aquí se inició todo!, teníamos el aparato locomotor, que según el Profesor Lain Entralgo «es una unidad biológica perfecta». Como ser vivo, desde un principio fue activo. Braus ve «en la actividad dentro del mundo la nota primaria de la función del cuerpo humano. Una fabrica en pleno rendimiento y capaz de moverse en el espacio».

En esta maquina humana, según él, «tenemos que admitir un para $q u e ́$, que nos permita describir con acierto una forma viviente, en la cual «TAL PARTE ES ASÍ Y HA LLEGADO A SER ASÍ PARA QUE PUEDA HACER LO QUE HACE, Y EN CONSECUENCIA DEBE HACERLO».

\section{El hombrecillo habilidoso}

En cumplimiento de ese «debe hacerlo», en una búsqueda instintiva del movimiento y del llamado bienestar psico-físico, la máquina humana sintió hambre y puso manos a la obra para calmarla, fruto de lo cual, dejó huella de sus habilidades para fabricar herramientas con las que procurarse alimentos, lo cual suponía desarrollar una habilidad para fabricarlas y utilizarlas. Nuestro hombrecillo habilidoso, jel habilis!, puso en marcha la actividad psíquica (jay el hambre, cuanto le debemos!) que originó la actividad física. El ejercicio de la decisión $\mathrm{y}$ de la voluntad operativa, se tradujo en MODIFICACIÓN ANATÓMICA Y FISIOLÓGICA DEL CUERPO. ¡Había nacido el concepto de entrenamiento! Esto en palabras de M. Harris fue el alba de la tecnología. Los individuos que fabricaban los mejores palos, hachas, lascas y las manejaban con mas habilidad disfrutaban de dietas más ricas en grasas y proteínas, serían más fuertes y sanos y dejarían mas descendencia. ¡El primer programa de entrenamiento, natural, estaba en marcha! 
La capacidad de aprender, almacenar y transmitir información depende de capacidades específicas determinadas genéticamente. Estos grupos de cazadores-recolectores disponían de un pequeño repertorio de tradiciones sencillas que se transmitían de generación en generación.

Eliseé Reclus, en su libro «L'Homme et la terre!, hablando sobre la aparición del deporte y la tendencia a jugar del hombre señala, «fue, después de la alimentación, la forma más antigua de la actividad de los hombres». La selección natural del mejor estaba en marcha, el mejor enseñaría al resto, en el tiempo libre, entre expediciones de búsqueda de alimento, perfeccionarían sus herramientas y su manejo, los jóvenes jugarían a imitar los gestos y luchas de sus mayores, idel mejor de sus mayores! Luego vino la defensa de sus territorios, de sus presas, etc. Llegó el grupo, la banda, la aldea, podemos decir «el equipo». Los hombres recibían entrenamiento para ser cazadores, proveedores de proteínas y grasas. Eran seleccionados para el papel de guerreros, cazadores de hombres, por su habilidad como cazadores de animales (Preparación militar y «deportiva»).

Si la fuerza, en todas sus formas de manifestación, les permitía procurarse comida y triunfar sobre las bestias o sobre sus enemigos, lo lógico es que se preocupasen de aumentarla (Braus manifiesta «su profunda creencia en la CAPACIDAD CREADORA DE LA VIDA BIOLÓGICA»).

Siguen varios autores analizando la vida de estos grupos de jóvenes, indicando que se reunieron, construyeron un lugar para vivir juntos y se sometieron a un entrenamiento ascético, que los preparaba para sus futuras expediciones, cuya finalidad no era desinteresada, puesto que debían desembocar en el rapto de jóvenes mujeres extranjeras.

Para Ortega y Gasset este entrenamiento y este agrupamiento, "primer club deportivo", han marcado la transición entre la horda y la tribu, anterior a la familia, y en ello ve lo que denomina «el origen deportivo del Estado».

Desde la misma aparición del hombre sobre la tierra podemos decir que la acción le ha acompañado. A través de ella ha podido ir solucionando sus necesidades de todo tipo. Ortega y Gasset ha diferenciado y mostrado de forma atractiva la oposición entre diversas actividades: «De este esfuerzo obligado, por la estricta satisfacción de una necesidad, el ejemplo típico es lo que el hombre llama comúnmente trabajo. Del esfuerzo superfluo, el ejemplo mas claro es el deporte. Este nos lleva a invertir la jerarquía secular y considerar la actividad deportiva como actividad primordial y creativa, la mas elevada, la mas seria y la más importante de la vida, y la actividad laboral como derivada de la primera, como simple decantación y precipitado de aquella. Mas 
aún, no hay vida hablando con propiedad, mas que la vida de aspecto deportivo, no siendo el resto, relativamente, otra cosa que mecanización y simple funcionamiento» (La Revista Europea).

\section{¿Hubo entrenamiento antes de los jefes?}

Es decir, ¿antes que entrenadores? Si, como acabamos de ver. Desde que el hombre apareció en la tierra se entrenó porque era necesidad vital. Hecho para la acción, y con necesidades psíquicas, físicas y técnico-motrices puso su potencial al servicio de sus necesidades, perfeccionó aquel y resultó más eficaz en la resolución de sus problemas. Todo esto se desarrolló en el marco de la selección natural y cultural. Las vivencias individuales aportaron experiencia a los individuos, que transmitieron a los demás. Sumó todas sus experiencias motrices y las fue trasladando de una actividad a otra, todo basado en su inspiración y capacidad creadora. ¡El primer «autodidacta obligado» había nacido!

\section{Que entendemos por entrenamiento}

La palabra entrenamiento viene usándose normalmente con múltiples significados. Los fisiólogos entienden como entrenamiento TODAS LAS CARGAS FÍSICAS QUE PROVOCAN ADAPTACIÓN FUNCIONAL Y MORFOLÓGICA, UN CAMBIO DE ORGANISMO Y, POR CONSECUENCIA, UN AUMENTO DE RENDIMIENTO.

En sentido más amplio hoy el concepto de ENTRENAMIENTO SE USA PARA TODO PROCESO FORMATIVO ORGANIZADO QUE TIENE POR FINALIDAD EL RÁPIDO INCREMENTO DE LAS CAPACIDADES FÍSICAS, PSÍQUICAS, ESPIRITUALES Y TÉCNICO-MOTRICES DE LOS INDIVIDUOS, y en el mundo del deporte, el proceso de preparación de los atletas a rendimientos altos y muy altos. El entrenamiento deportivo es el proceso de perfeccionamiento guiado por principios científicos, particularmente pedagógicos, que, incidiendo sobre la capacidad de rendimiento y sobre la disponibilidad de cada atleta, trata de conducirlo a altos rendimientos en una o más disciplinas deportivas.

LA FUNCIÓN DE TODO ENTRENAMIENTO DEPORTIVO CONSISTE EN LA PREPARACIÓN PARA LA COMPETICIÓN. 


\section{Ciencia y entrenamiento}

«La simiente de la ciencia es el deseo de saber. Pero cada paso en el sendero del saber es un misterio. A comenzar por el sendero mismo. El cual no se abre en todas las almas, aunque en todas existe. Hay seres para quienes el deseo de saber no se sabe a sí mismo, y en cuanto a los demás, hay lo menos tres colores de senderos: EL QUE DESEA SABER PARA HACER, el que desea saber para saber, Y EL QUE DESEA SABER PARA JUGAR. Del primero salen las artes de la política $y$ de la guerra; del tercero salen el amor y todas las artes, útiles, malas o bellas, así como el deporte; solo del segundo nace la ciencia. Porque la ciencia es el puro saber desinteresado. Que luego haya quien haga fructificar el saber, desde luego; pero el impulso prístino de saber es en si su propio fin y recompensa». Esto nos dice Don Salvador de Madariaga, y es mas que suficiente.

El entrenamiento perteneció en un principio a ese apartado que el gran pensador dedica a los «seres para quienes el deseo de saber no se sabe a sí mismo». La supervivencia era su maestro.

En la actualidad pertenece claramente al primer y tercer sendero que el maestro señala: al mundo que desea saber para hacer y al que desea saber para jugar, aunque cada vez se impone el primer sendero, del cual salen las artes de la POLÍTICA y la GUERRA. La competición deportiva actual de todos los niveles, sustituye a la guerra y es empleada cada vez mas por la política.

\section{¿Cuándo se inició el entrenamiento sistematico?}

¡El mismo día de la primera batalla de dos ejércitos organizados!

La fuerza física del hombre y su habilidad en el manejo de las herramientas-armas o maquinas de guerra fue decisiva durante muchos miles de años, y por tanto su entrenamiento fue abordado como preparación militar, y en tiempos de paz, se competía como medio de mantener el potencial de guerra del ejercito y distracción. De la eficacia de su ejercito dependía la existencia de un país o ciudad, podemos suponer que todos los conocimientos de los que componían la sociedad se empleaban en el diseño de la preparación de su ejército. Los conocimientos científicos de todas las culturas eran la base del entrenamiento de su ejército, y los entrenadores... los jefes de dicho ejército.

Egipcios, Asirios, Babilonios, Medo-persas, Celtas, Griegos, se impusieron en el mundo gracias a su cultura y.. a sus ejércitos espe- 
cialmente organizados, preparados y entrenados. Las experiencias se transmitían, se eliminaban errores, en fin, el método deductivo (ciencia). De estas culturas, y su relación con el ejercicio físico y la competición deportiva, sabemos a través de sus artistas, que escribieron, esculpieron, pintaron, etc., dejándonos el testimonio de su quehacer diario.

Son famosas las competiciones atléticas de los egipcios y ciertas comarcas de Asia Menor. Eran sobre todo carreras y saltos, con equipo militar, naturalmente, ide que nos sirve un gran saltador si solo salta mucho cuando va desnudo!

También antes de nuestra era, en Irlanda se celebraban los Tailtin Games, existían desde el siglo XIX a.C., se celebraron durante veinticinco siglos. La competición tenía entre otras, un salto de altura que formaba parte de la preparación militar gaélica. Para formar parte del ejercito era necesario ser capaz de saltar por encima de un hombre de pie. El lanzamiento era el «roth cleas», el ancestro lejano del martillo.

Podemos decir, que cuando una cultura establece competiciones de algún tipo, estas corresponden a alguna finalidad (fiesta, culto, conmemoración, funerales, etc.), que dan prestigio social, promoción social, beneficio material, etc. o todo a la vez, a quienes en ella destacan. Teniendo esa repercusión social y material podemos asegurar que el entrenamiento para ellas fue objeto de reflexión y estudio en todas las épocas.

\section{¿Qué bases tenía el entrenamiento de nuestros antepasados más remotos?}

a. La alternancia TRABAJO-RECUPERACIÓN. Obligados por el ciclo circadiano, alternaron actividad con sueño, por mucho hambre que tuvieran, la fatiga les obligaría a descansar. Poco a poco experimentaron la supercompensación.

b. Emplearon «ejercicios específicos» para prepararse. Solo la práctica con la propia herramienta-arma les daba la habilidad para la "competición» (jéxito en la caza mayor o menor, para alimentarse o vencer!). No había "preparación general», todo era específico.

c. Empíricamente conocían las dos bases del entrenamiento: minimizar el gasto y maximizar la potencia disponible.

d. ¡La selección de talentos! Estos si que sabían detectarlo, sin necesidad de procedimientos de laboratorio. Los mejores comían mas veces y mejores cosas... fruto de su verdadero talento. 
Posiblemente solo en estas etapas de la vida del ser humano se empleó un medio de entrenamiento que en la actualidad no es fácil de emplear. Las largas y quizás frecuentes, dietas involuntarias, durante los días de escasez de recursos alimenticios, mas bien su carencia total durante largos espacios de tiempo, SERVÍAN DE ENTRENAMIENTO AL ORGANISMO PARA MEJORAR SU EFICACIA COMO MÁQUINA ENERGÉTICA.

\section{¡Los griegos!}

La actividad atlética fue preocupación de los griegos durante casi dos mil años, desde los juegos fúnebres en honor de Patroclo hasta el edicto de Teodosio suprimiendo los juegos atléticos.

$\mathrm{Su}$ origen y «presidencia de honor» pertenecían a un dios, como no podía ser menos en un lugar donde un poeta de aquellos tiempos se lamentaba: «No se sabe ya donde esconder una fanega de trigo: ¡cada hoyo está ocupado por un dios!». Ya en «tierra», los de a pie también han sido objeto de observación por ilustres escritores.

«Miles de hinchas acudían a lo largo de las siete carreteras que conducían a Olimpia, camino arbolado de Argos hasta el rio Alteo discurría entre templos, estatuas, tumbas y bancales de flores. Podían encontrarse en él, del brazo, a diputados de izquierda atenienses y generales espartanos, e incluso grupos de filósofos en paz entre ellos». Así describe la «situación» Indro Montanelli (Historia de los Griegos), ¡vamos, como si hablase de hoy mismo, de los alrededores del Nou Camp o del Bernabeu!

En cuanto al asunto que nos ocupa, el entrenamiento, podemos asegurar que en la actividad deportiva griega todo era profesional. Los técnicos, de muy diversas formas denominados a través de esos casi dos mil años y de los diversos lugares geográficos, tenían formación académica y misiones perfectamente definidas. Todo lo relacionado con la actividad física, educación o competición, estaba en manos de equipos de profesionales.

Entre los griegos, el verdadero objetivo de las competiciones deportivas era triunfar sobre los adversarios, jafortunadamente para ellos no existían los récords!, ¡«Grecia es ante todo el país de la inteligencia, y la astucia no es mas que una forma de la inteligencia. Tanto peor para el atleta que no tiene mas que su fuerza»!

«Un griego no ve triunfar con placer al hombre dotado de medios físicos que le dispensan de reflexionar, de calcular, de combinar. En la guerra el que engaña al enemigo es un gran general; ¿por qué 
debería ser diferente aquí? No se admira menos a Ulises por ser el hombre de las astucias múltiples que a Aquiles por ser el hombre de los pies ligeros».

Había deslealtades hacía las reglas, y era mas un combate que un juego.

Los griegos no fueron solo filosofía, búsqueda de los grandes por qué de la vida y del universo, también se dedicó al estudio del «como», que según dicen los grandes pensadores, es el cometido de la Ciencia. $\mathrm{El}$ «como» del deporte y el entrenamiento entre los griegos, fue amplia y profundamente estudiado, en un rápido vistazo a los pocos escritos que nos han llegado, vemos que lo más selecto de casi dos milenios se ocupó del asunto:

HIPOCRATES: primer médico laico, antes solo los sacerdotes podían curar. Higienista. En su Tratado de la buena higiene, analiza la deshidratación del deportista, el valor del frío (polvo) y el calor (aceite). Valora la importancia de la dieta para el deportista.

JENOFONTE: Tratado de la Fatiga y Tratado del Sudor.

ARISTÓTELES: en su «Política» trata del entrenamiento individualizado, de la importancia de la herencia (genética) en los resultados deportivos, así como la antropometría, biotipo y temperamento, especial para cada deporte. Analiza la diferencia entre lanzar con la mano vacía y con una piedra (jconcepto de velocidad gestual!). Los calambres después de la carrera. El efecto del entrenamiento sobre la grasa del cuerpo del deportista.

ESTRABON: en su Geografía. Los diferentes lugares y su clima influyen sobre la salud y la forma atlética. Su frase "mas sano que Crotona».

PLUTARCO: «Philopoemen». Analiza la diferencia entre la preparación militar y el entrenamiento atlético. "Los atletas aumentan y mantienen su forma durmiendo mucho, con la sobrealimentación, ejercicios y pausas cuidadosamente regladas... Los soldados por el contrario deben someter su cuerpo a las circunstancias más diversas: por encima de todo deben endurecerse para prescindir de alimentos, de bebida y de sueño».

GALENO: aunque sentía algo de desprecio por la gente del deporte y sus excesos, escribe sobre el ejercicio mas adecuado en cada edad y a cada temperamento, y como no podía faltar en un médico, elogia los juegos de pelota (jexentos de violencia y llenos de «buenas maneras» en la época! Pero en su Discurso sobre las artes sale al ruedo y... "Ahora bien, la forma del atleta llevada a su plenitud es un estado precario y fácilmente modificable. Una cosa que ha alcanzado su plenitud no es susceptible de aumentar, no puede permanecer en el mismo estado ni pararse: no puede mas que declinar». Y no nos defrauda 
su inteligencia cuando en su "De la protección de la salud", lib II cap IX-XI analiza con gran profundidad y agudeza las cualidades físicas del hombre: «la fuerza», «la velocidad», «fuerza y velocidad», los «juegos de músculos». Y en sus Conclusiones: «En resumen, el entrenador experto en su arte, debe conocer todos los deportes y llevar cada uno de ellos a su fin propio». Mas tarde se muestra partidario del entrenamiento «muy específico».

Los testimonios se prolongan en la ANTOLOGIE DES TEXTES SPORTIFS DE L'ANTIQUITÉ (1927) y otros textos de poetas e historiadores. La pregunta se impone, ¿qué conocían los griegos del entrenamiento deportivo? En mi opinión... TODO lo que hoy consideramos básico, para adaptarse, es decir entrenarse. Conocían todos los principios del entrenamiento.

- Carga de entrenamiento, está en casi todas sus recomendaciones.

- Progresividad de la carga, aquí tenemos que recordar a Milon de Crotona, campeón olímpico de lucha en seis ocasiones (Olimpiadas números 60-62-63-64-65-66, a lo largo de veinticuatro años 540-516). Cada principio de año olímpico compraba un joven novillo y luchaba diariamente con él. El novillo crecía e iba incrementando su fuerza... lo mismo que su oponente. Un prodigio de aplicación del principio de progresividad de la carga de entrenamiento y del de frecuencia de entrenamiento. Si dejaba de luchar algún día, su adversario tomaría ventaja.

- Variedad de la carga, existen recomendaciones para corredores de variar la intensidad, la técnica de carrera, la superficie de carrera, el perfil (cuestas y descensos), trabajo con lastre, luchas con diferentes adversarios, con el cuerpo engrasado, trabajo al aire libre y a cubierto, en todo tipo de climas y de horario, etc.

- Periodización del entrenamiento, es decir, división a medio plazo del entrenamiento en periodos con objetivos diferenciados y, por consiguiente, con contenidos de entrenamiento y métodos de entrenamiento diferentes, en particular cargas de entrenamiento distintas.

Los griegos iniciaban la preparación para Olimpia diez meses antes (¡macrociclo!) y un mes antes los participantes se reunían en Elis (¡mesociclo de preparación a la competición!) y por si faltaba algo, describen con todo lujo de detalles un microciclo de cuatro días que llamaban TETRADA, de la siguiente forma:

$1^{\text {er }}$ día.-se prepara al atleta. El trabajo es BREVE, ENÉRGICO y RÁPIDO.

$2^{\circ}$ día.-es de actividad intensa del atleta definido por la «potencia de trabajo». 
$3^{\text {er }}$ día.-descanso del atleta. Trabajo ligero, masaje, baño, reposo.

$4^{\circ}$ día.-esfuerzo medio del atleta. Trabajo de estrategia y táctica de su especialidad.

¡Este sistema de TETRADAS fue combatido por FILOSTRATOS, porque se limitaba la iniciativa del entrenador y no tenía en cuenta las condiciones climáticas!

Conocen con gran profundidad las cualidades físicas básicas: fuerza, en todas sus manifestaciones, velocidad (que valoran sobre todas las demás), resistencia, elasticidad, coordinación y los medios y métodos para desarrollar cada una de ellas. Dominan la enseñanza de las técnicas que describen en tratados específicos. Hablan del calentamiento previo y vuelta a la calma. La programación de la actividad competitiva y del entrenamiento es habitual, existiendo profesionales en todos los niveles. Los medios de recuperación biológica son conocidos y aplicados con normalidad en el entrenamiento y las competiciones. Respecto a la preparación biológica, es mencionado con naturalidad y podemos afirmar que sin limites para los conocimientos de la época en este campo, que no debían ser pocos a juzgar por el refinamiento de vida. ¡Casi contemporáneo de ellos, Obelix, el gran proveedor de rocas y voraz perseguidor y depredador de jabalíes, recurría a su druida (¡médico deportivo!) favorito para la pócima mágica (¡preparación biológica!). El nombre del famoso druida no deja mucho campo a la imaginación: ¡MIRACULIX! ... también llamado Panoramix. No faltaba el «espía industrial»... por nombre Detritus.

\section{¿... Y hasta hoy?}

Poca cosa. Los tratados de los renacentistas,

MERCURIALIS:1530-1606 Italia ARTE GIMNÁSTICA, influido por científicos italianos (B. PLATINA, G. BATTISTA MONTE, C. CARDOSO, A BOTTONI). Esta obra tiene tres partes:

Gimnasia Higiénica

Gimnasia Militar

Gimnasia Atlética

RABELAIS: 1494-1553. Gargantua y Pantagruel. Gargantua es el ancestro del decatleta actual: corre, salta, pasa obstáculos, lanza piedras y jabalina, tira al blanco con el arco, y no olvida la fuerza que desarrolla con halteras, trepa, etc. ... y se relaja con la natación y hace el Tarzan gritando para desarrollar su tórax. ¡Completísimo... aunque algo rústico! con relación a los griegos. 


\section{Bases científicas del entrenamiento deportivo}

MontAignE: 1583-1592, en sus EsSAIS. Como ha leído a Platon, nos recomienda correr, saltar, cabalgar, bailar y la música... y ser resistentes.

El siglo XIX es más interesante, y el humanismo inglés inicia la metodización del entrenamiento y sistematizan el trabajo. Norteamérica se une al movimiento en la última parte del siglo y surge el concepto de intervalo en el entrenamiento de corredores que llaman TEMPO TRAINING. También hay algunas obras sobre el entrenamiento, DU FOUR escribe AGONISTICON.

En general, los estudios sobre el entrenamiento se centran en los sistemas de entrenamiento para corredores.

Tengo que confesar, llegado a este punto, mi falta de entusiasmo por todos estos siglos, hablando de entrenamiento y ser humano, ilos griegos dejaron el tema agotado, y, además, sus artistas en su afán de correr en socorro del vencedor (igran protagonista del acontecimiento humano más importante de la época!) maquillaban lo visto y oído.

«Poseedor de lo más valioso para los griegos, la victoria, «la corona»!, Para arrancar esta victoria los atletas prodigaban esfuerzos violentos, los corredores se crispaban por la fatiga, el boxeador se agota por el dolor, y no podían conservar esta actitud tranquila y relajada en la cual han sido inmortalizados. Los escultores, para expresar el perfecto equilibrio y la superioridad del vencedor, han idealizado sus modelos».

Poco se pudo hacer durante siglos.

\section{El entrenamiento moderno}

Los sistemas de entrenamiento creados sobre apoyo científico aportado por las ciencias biológicas, físicas y psicopedagógicas, comienzan a finales del XIX y principios del XX, con la Escuela Alemana de GuTs MUTHS.

El concepto biológico del entrenamiento como adaptación específica a un esfuerzo es enunciado por Wilhelm Roux, con su Teoría biológica «de la excitación funcional» creando una expresión ya familiar de que «la función crea el órgano».

En 1881 expone el enunciado: «una exigencia más intensa de las capacidades orgánicas produce una alteración de las mismas en el sentido de elevar específicamente su capacidad de rendimiento». Este enunciado expuesto para cualquier tipo de organismo constituye la base científica del entrenamiento deportivo.

En 1877 Arndt expone la Teoría de la integración entre estímulos y la materia viva. «Estímulos suaves excitan levemente las funciones 
orgánicas. Estímulos fuertes producen fenómenos de adaptación y estímulos demasiado fuertes producen daños en el organismo que inclusive pueden llevar a la muerte».

Mas tarde SELTE H. (1907-1982) expone su teoría del estrés y el síndrome general de adaptación, por el cual un organismo reacciona a todo tipo de agresiones.

Redondea las bases biológicas del entrenamiento Uthomskij, añadiendo: «El organismo restituye en un lapso de tiempo corto las energías gastadas por el esfuerzo y posteriormente desarrolla una mejora energética para encontrarse mejor capacitado ante sucesivos esfuerzos, denominando a este último proceso de RESTITUCIÓN AMPLIADA. El conocimiento de este fenómeno será de vital importancia para que los estímulos posteriores sean aplicados en el momento en que el organismo haya completado su proceso de restitución para no caer en la fase de agotamiento».

El concepto biológico del entrenamiento plantea el conocimiento exacto de la dosificación del volumen y de la intensidad del entrenamiento, su momento de aplicación y los efectos fisiológicos producidos sobre el individuo a quien lo estamos aplicando.

Uno de los primeros sistemas de entrenamiento basado en principios biológicos es el «intervall training», creado por Woldemar GERSCHLLER y el Prof. RAINDELL, de Friburgo, en 1940-1950. Poco antes, en 1939, el ruso K. GRANTYN propone el primer sistema que consta de un ciclo anual completo de entrenamiento, sin interrupciones, con periodos de entrenamiento más precisos de lo que se había hecho hasta la fecha.

Sucesivamente, N. OZOLIN y S. LETUNOV (1950) hacen puntualizaciones al programa de Grantyn y llegamos así a 1956, año en el que con la propuesta de la PERIODIZACIÓN ANUAL DEL ENTRENAMIENTO de L. P. MATVEEV, y fecha en la que nace la VERDADERA CIENCIA MODERNA DEL ENTRENAMIENTO.

¿Qué cambió con MATVEEV?

Por fin hubo una estructura completa del entrenamiento prolongado durante todo el año. Articuló en fases adaptativas la FORMA DEPORTIVA del atleta y la ORGANIZACIÓN PEDAGÓGICA de la CARGA PARA HACER COINCIDIR LA FORMA Y EL CALENDARIO DE COMPETICIONES.

¡Por fin íbamos a saber cuando estaríamos en forma, y esta coincidiría con la fecha de las competiciones más importantes!.

L. P. Mateev se encargó de organizar los programas de entrenamiento de la antigua URSS a partir de la incorporación a los JJ.OO de Helsinki de este país y se inició la «carrera científica» hacia la obtención de resultados deportivos. El prestigio que estos dan a todo tipo de pro- 
fesionales, hace que a partir de los años 50 se suceda una verdadera "pirotecnia terminológica» de la diferenciación de pequeños matices en los sistemas de entrenamiento.

A. Arosjev en 1971 «inventa» el principio del entrenamiento en PÉNDULO, apoyándose particularmente en la idea del contraste de los efectos del entrenamiento sobre el organismo del atleta: UNA ALTERNANCIA SISTEMÁTICA (PENDULAR) ENTRE CARGA ESPECIAL Y GENERAL, en la que la CARGA ESPECIAL, a diferencia de la general, CRECE SIEMPRE MAS, HASTA ALCANZAR SU CULMINACIÓN CON EL PERIODO DE COMPETICIÓN.

A. Vorobjev, entre 1971 y 1977, aporta el concepto de «cambio a SALTOS» de la INTENSIDAD y DEL VOLUMEN DE LA CARGA DURANTE TODO EL CICLO ANUAL. Y las cargas deben cambiar incluso en sus contenidos (tipo de trabajo y tensión muscular, etc.).

Verchoshanskij, entre 1979 y 1985 desarrolla con éxito su ESTRUCTURA DE BLOQUES DE ENTRENAMIENTO.

La base de su sistema es la CONTINUA INTENSIFICACIÓN DE LA CARGA A TRAVÉS DE LA PREPARACIÓN CONDICIONAL ESPECÍFICA. Es ir directamente al control de la adaptación del aparato motor del atleta a la carga específica de competición.

Concentrando los contenidos sobre determinadas estímulos entrenantes crea bloque de fuerza específica, de técnica, de velocidad.

Durante todos estos años se suceden las luchas entre facultades, escuelas, terminología y «nuevas versiones» en todo lo que se refiere al entrenamiento y a la teoría del entrenamiento deportivo.

\section{Tendencias actuales de la teoria del entrenamiento}

En la literatura actual de la metodología deportiva se encuentran todo tipo de opiniones sobre el sistema de entrenamiento deportivo, así como concepciones y escuelas diversas de preparación de los deportistas, y esto es natural y lógico tratándose de un fenómeno tan multiforme como es la actividad deportiva.

En las tendencias actuales han influido e influyen constantemente, los progresos científicos de la fisiología y de la BIOQUÍMICA de la actividad muscular, de la MEDICINA DEPORTIVA, de la BIOMECANICA de los gestos deportivos, y los estudios básicos en la metodología del entrenamiento en el deporte de alto nivel. Todo esto ha creado nuevos supuestos objetivos para la formulación de la moderna teoría y metodología del entrenamiento, y sus principales bases científicas. 
Pero conviene no olvidar otros factores que han influido, y muy decisivamente, en los cambios de esa teoría y metodología del entrenamiento.

A tener en cuenta en primer lugar los cambios en el deporte internacional, en particular la intensificación del calendario de competiciones, que ha cambiado completamente ritmos y tiempos de la preparación poniendo sobre nuevas bases el problema de la CONSECUCIÓN y MANTENIMIENTO del estado de FORMA.

Como consecuencia de esta transformación está el significado o valor, en algunos aspectos nuevo, que han adquirido las COMPETICIONES EN EL CUADRO DEL ENTRENAMIENTO, a corto y largo plazo, de los atletas.

Otro acontecimiento a tener en cuenta ha sido la activación de la LUCHA ANTI DOPAJE, y el final de una cierta «impunidad» de la cual habían gozado los atletas de algunos países. Aunque el problema dista mucho de estar resuelto del todo, no hay duda que esto ha tenido su influencia en el campo de la teoría y metodología del entrenamiento y ha puesto a discusión volumen e intensidad de trabajo, periodización, etc.

A esto hay que añadir los cambios habidos en algunos países con el DESCUBRIMIENTO (o casi) del ATLETA de ESTADO. Esto es una figura, objeto, mas que sujeto, de planificación y estructuras de entrenamiento posible, dado que está completamente disponible y dependiente de la organización deportiva de forma total.

Lo mas «à la page» en cuestiones de entrenamiento es «la prioridad del aspecto biológico en la teoría del entrenamiento».

Investigadores como Platonov (88), Nikitjuk, Samoilov (90), Voss (91) Tschiene (91) y otros, orientando sus trabajos a la adaptación de los diversos sistemas de órganos del atleta de alto nivel, confirman que en la teoría del entrenamiento, es cada vez más dominante el aspecto biológico.

En la practica del entrenamiento, todas las aportaciones de las llamadas ciencias auxiliares del entrenamiento, que son muchas, nos están llevando a una nueva situación, en la cual cada vez sabemos mejor «a dónde vamos», es decir, lo que queremos conseguir, y en consecuencia podemos seleccionar mejor los «vientos favorables», los medios con los cuales conseguiremos el objetivo de llegar al lugar que «ya casi conocemos».

En su trabajo «Fundamentos de una nueva teoría del entrenamiento para el deporte competitivo», S. Zanon (92) nos dice: «El entrenamiento para el deporte competitivo consiste en secuencias de comportamientos bien organizados y biológicamente finalizados, que actúan según una 
integración temporal con carácter micro y macro cíclicos. La adaptación solicitada en el entrenamiento para el deporte competitivo, aunque con carácter cíclico, es siempre realizada a través de la intervención de mensajeros bioquímicos, cuya acción implica la variación del metabolismo a través de la unión del mensajero con el receptor correspondiente, a nivel celular. El mensajero bioquímico y el correspondiente receptor pertenecen a los tres grandes sistemas reguladores del organismo, denominados Sistema Nervioso (SN), Sistema Endocrino (SE) y Sistema Inmunológico (SI). Por eso el entrenamiento para el deporte competitivo es siempre y únicamente un comportamiento adaptativo orientado a obtener una nueva expresión del metabolismo, a través de una variación del número de los receptores en la célula».

Estudios en el área de la actividad muscular, han llevado a un mayor conocimiento del concepto de «ENERGÍA ELÁSTICA» en los músculos humanos y su aprovechamiento, así como en la unidad músculos, tendones, huesos y ligamentos. En realidad todo el cuerpo es estudiado como un acumulador de energía elástica.

\section{Características de la práctica del entrenamiento hoy}

Durante toda la historia de la teoría y práctica del entrenamiento, la gran interrogante ha sido qué medios son más eficaces para conseguir adaptarse a las exigencias de la competición, es decir, para rendir mas, llegar a los límites genéticos de cada individuo, naturalmente en la especialidad para la cual se entrena.

Los medios de entrenamiento se dividen en dos grandes categorías:

a. PREPARACIÓN CONDICIONAL GENERAL: consiste en un entrenamiento general que NO ACTIVA LOS SISTEMAS DEL ORGANISMO (nervioso, metabólico, psicológico) de un modo similar a como son activados en competición.

b. PREPARACIÓN CONDICIONAL ESPECÍFICA: activa los mecanismos físicos y mentales de forma extremadamente similar a lo que ocurre en competición.

Estos dos conceptos, entrenamiento general y entrenamiento específico, han estado en lucha durante todo este siglo. La variación del porcentaje de trabajo anual en cada uno de ellos ha ido variando y dando ocasión a «nuevos» sistemas, nombres, escuelas, etc.

Algo parecido podemos decir de los famosos principios de entrenamiento: el de la progresividad, el de la frecuencia y un largo etc. que variaba su importancia según los autores. 
Pues bien, podemos decir que hoy en el mundo del entrenamiento de deportistas de alto nivel, lo que predomina es el principio de la ESPECIFICIDAD, es decir, solo los estímulos específicos crean adaptación específica, solo los ejercicios que se parecen al de competición entrenan para la competición.

Este «parecido» de los ejercicios específicos de competición, es una alta correlación con el propio gesto de competición en sus NECESIDADES METABÓLICAS, BIOQUÍMICAS, ESTRUCTURALES, NEUROLÓGICAS, NEUROMUSCULARES, ETC.

En relación a la otra cuestión tradicional ¿VOLUMEN de trabajo o intensidad de trabajo?, la conclusión es tajante, se trabaja en INTENSIDAD TODO el año, SÓLO varia el volumen, mayor o menor según la proximidad de la competición.

¿Y que es la intensidad de trabajo? Aplicando el principio de la «especificidad», se considera trabajo específico solo aquel que va del $90 \%$ de la capacidad de rendimiento, al $100 \%$ (rendimiento máximo en competición). Si lo trasladamos a un deporte colectivo como el fútbol, podemos decir que solo cuando la exigencia del juego lleva al jugador a las 170-180 pulsaciones por minuto, está trabajando (creando estímulos de adaptación) con un ejercicio específico.

El aumento de la INTENSIDAD del entrenamiento y la ESPECIFICIDAD del mismo en todos sus aspectos, exige un aumento de los medios de regeneración biológica, a mas descansos, y mayores riesgos de lesión.

\section{De Atlanta a Sidney}

Como hemos visto anteriormente, existen distintos «pasos metodológicos», en el entrenamiento en aquellos países que disponen de conocimientos... y dinero para ello, particularmente lo primero en mi opinión. Ponen en práctica el $6^{a}$ paso, (ver gráfico 1) compuesto de profesionales de muchas ciencias trabajando en equipo. Se analizan los resultados de las competiciones más importantes a nivel internacional, el entrenamiento de los mejores atletas, de los propios atletas y su entrenamiento, se evalúa, se toman decisiones sobre el entrenamiento y aplicaciones metodológicas, se definen objetivos del entrenamiento y programación del mismo, para las próximas temporadas o ciclos olímpicos.

Uno de esos países, Alemania, a través de su Instituto para la ciencia aplicada del entrenamiento de Leipzig, realizó un análisis de los JJ.OO de Atlanta desde el punto de vista de la Teoría del entre- 
namiento, y las consecuencias que se obtienen para la nueva orientación del sistema de deporte de alto nivel.

En este tipo de estudios se buscan las tendencias, los elementos que caracterizan las prestaciones de alto nivel internacional, que pueden ser retenidas como validas para un grupo de deportes.

Se señala también el desarrollo de los sistemas de entrenamiento y de competición a estos niveles. Evaluando el ciclo olímpico 92 al 96, de Barcelona a Atlanta, figuran en su informe estas observaciones:

- «Existen diferencias en la evolución de los resultados según deportes, que van desde el progreso en algunos deportes a la regresión en otros».

- «El número de los atletas/de los países, capaces de obtener medallas va en aumento. Existe un gran paralelismo entre el elevado ritmo de la mejora de los resultados y el aumento de la profesionalidad y comercialización. Actualmente sin una preparación de tipo profesional no es posible realizar prestaciones de nivel mundial».

- «Los atletas de clase mundial realizan cada vez mas su preparación como una profesión. Son profesionales en el sentido más amplio del término, y gracias a estructuras igualmente profesionales, del sistema competitivo y de entrenamiento del deporte por ellos practicado, les garantiza un entrenamiento eficaz y una organización de las competiciones orientada a prestaciones de alto nivel. En aquellos deportes caracterizados por un alto grado de internacionalidad y de comercialización, solo profesionales pueden crear nuevos criterios en la implantación y en la carga del entrenamiento».

- «Las ventajas sobre todo sociales y financieras ligadas a la victoria, a la medalla, a los récord, a los resultados espectaculares, están siendo utilizadas aún mas que en el pasado como estímulo al éxito, especialmente por los atletas de los países en vía de desarrollo y de los países del ex-bloque oriental, siendo el resorte que desencadena una mayor disponibilidad agonística y al riesgo, ya sea competición o entrenamiento".

- «Los desarrollos, de repente, de los resultados surgen como fondo de los efectos de los muy diversos sistemas de los controles antidopaje que son muy distintos entre deportes:

- en lo que concierne al número de atletas afectados por ellos

- el procedimiento empleado

- los momentos de los controles durante el año, sobre todo de los controles por sorpresa durante el periodo entrenamiento.

En estas condiciones se puede afirmar que actualmente no está garantizada la plena igualdad de oportunidades de los atletas que participan en la competición». 
- «En aquellos deportes en los cuales encontramos un ingreso precoz en la edad de los máximos resultados tienen ventajas aquellos países que han desviado en favor del entrenamiento un esfuerzo global representado por la escuela y el entrenamiento. Esta es también una condición que no garantiza ya la igualdad de oportunidades de los atletas que participan en estos deportes».

- «Una de las tendencias mundiales más importantes que se pueden observar es el elevado grado de internacionalización de los sistemas de entrenamiento (...) se observa en países que tienen tradicionalmente un peso en el deporte de alto nivel (Francia, España, Suiza) pero también en estados con cotas mas elevadas de inmigración (Canadá, Australia, Nueva Zelanda, etc.) donde se pueden observar notables esfuerzos hacia una transformación de la orientación de los sistemas de entrenamiento, que se basan, entre otras cosas, también en la utilización de entrenadores e investigadores de los países del ex-bloque oriental y sobre la asimilación de conocimientos de la ciencia del entrenamiento».

Estas y otras «perlas» se encuentran en la primera parte del estudio del IAT de Leipzig.

En su segunda parte, analizando los bajos resultados nacionales y sus causas, señala las siguientes:

1.-La falta de un continuo acceso de nuevas «quintas» de atletas del sector de "promesas» al sector de "primera línea».

2.-El excesivo retraso en la especificación de cuales son las tendencias internacionales de desarrollo con el consiguiente retraso en la reacción a ella del sistema de entrenamiento.

3.-Excesiva y prolongada fidelidad a los programas de entrenamiento existentes.

4.-Errores en la periodización del entrenamiento.

5.-Una valoración funcional (diagnosis del entrenamiento) que toma en consideración o parámetros no específicos para el deporte considerado o demasiado pocos parámetros llevando a conclusiones erróneas.

6.-Interés excesivo por las competiciones nacionales por parte de los clubes deportivos o de los propios atletas, que entra en conflicto con la concentración de preparación del equipo nacional.

Creo que podemos suscribir el diagnóstico para nuestro país en todos los apartados. Limitándonos a nuestro objetivo, el entrenamiento y su evolución, los expertos de Leipzig, Dietrich Martin, Jürgen Krug, Manfred Reib y Klaus Rost, señalan como características de los modernos sistemas de entrenamiento y competición lo siguiente: 
Las tendencias más importantes en los sistemas de competición son:

- El aumento del número de competiciones internacionales importantes. Que influye sobre la construcción de la prestación durante el entrenamiento anual y a largo término.

- El creciente aumento del peso de la «comercialización» que refuerza tanto la aspiración a récord y a victoria como a resultados espectaculares por parte del atleta, por lo cual aumenta su disponibilidad a competir y a arriesgar, sobre todo en aquellos deportes que interesan mas a los medios de comunicación.

- A esto se contrapone la evidente diversidad en lo que a eficacia se refiere entre los programas de competición del sector del deporte de elite y el juvenil.

Esto en cuanto a la acción y la influencia de los sistemas de competición sobre los sistemas de entrenamiento ligadas a ellos.

Tendencias más importantes en lo que a sistemas de entrenamiento se refiere.

El aumento de la eficacia del entrenamiento mismo, es la consigna internacional según ellos, y añaden las siguientes tendencias:

1.-Adaptación del entrenamiento al número creciente de competiciones, con consecuencias sobre la estructura de la prestación, sobre la de la carga y de la construcción del entrenamiento anual.

2.-Ampliación de las particularidades del entrenamiento con cargas al limite de la capacidad individual de prestación, con consecuencias sobre la dinámica de la carga y la regeneración (trabajo-descanso).

3.-Una orientación cada vez mayor del entrenamiento hacia las futuras exigencias de competición. Según lo cual se debe plantear el entrenamiento no sobre la exigencia del nivel actual de resultados internacionales, sino sobre aquellos previsibles en el futuro.

4.-Aumento de los porcentajes de entrenamiento con el fin de garantizar la capacidad de carga, con el consiguiente empleo de métodos específicos e individualizados.

5.-Garantizar la eficacia del entrenamiento durante la construcción plurianual de la prestación, para lo cual los estímulos de la carga son fijados con arreglo al nivel creciente de adaptación.

6.-Utilización de las competiciones como medio principal para la formación de la prestación, con el consecuente aumento de los estímulos específicos de la carga.

Los «aruspices» de Leipzig resumen esta parte de su informe señalando las condiciones para llegar al aumento de la eficacia del entrenamiento:

a. Garantizar un elevado volumen de entrenamiento. 
b. Construcción de una «cadena operativa» sistemática en el sistema de competición y de entrenamiento.

La primera no es ninguna novedad, veamos qué es la segunda.

\section{La «cadena operativa»}

«La "cadena operativa" del sistema de entrenamiento y de competición parte de las características, de la estrategia, de las soluciones técnicas, de la interpretación de las reglas, de la tecnología de los artefactos y de las causas que condicionan el entrenamiento de las prestaciones internacionales de punta, y de su tendencia de desarrollo.

Los resultados internacionales de punta se presentan como prestaciones de estructura compleja. Cuanto más se consigue analizar esta estructura compleja, más exacto será el contenido de las informaciones sobre las condiciones que han permitido obtener estas prestaciones.

Por este motivo, el análisis de las competiciones tiene como objetivo aportar un cuadro lo mas objetivo y referido posible de cuanto ha ocurrido realmente, del cual se puedan obtener, sobretodo, objetivos de entrenamiento a corto y largo plazo. Las actuales condiciones de objetividad y de cientificidad ofrecen la posibilidad de un análisis similar de las competiciones.

Sigamos ahora con la «cadena operativa»: la concepción del entrenamiento, es decir, el esbozo del plano de entrenamiento ya constituye el programa de su realización, se obtiene de los objetivos, de las experiencias, de los índices internacionales obtenidos sobre el entrenamiento, de los modelos específicos de periodización y de las condiciones individuales y materiales generales, teniendo en cuenta las reglas fundamentales que determinan el entrenamiento.

Llegado a este punto, la «cadena operativa» funciona en general sobre la base del alto nivel de experiencia de una comunidad de entrenadores muy evolucionada.

Sin embargo, la segunda parte de la «cadena operativa» necesita que al lado de un entrenamiento directo de un entrenador haya un sistema de investigación que permita una interpretación científica aplicada, con orientación práctica, que hemos definido como «investigación sobre el entrenamiento y la competición que acompaña al proceso» (de entrenamiento $y$ competición).

Tal sistema de investigación requiere métodos de evaluación funcional con finalización específica, combinada con procedimientos de entrenamiento en centros dotados de instrumentos de medida, orientados según los criterios del modelo de la prestación de competición de nivel 


\section{Bases científicas del entrenamiento deportivo}

internacional. Son importantes sobretodo la objetivación y la exigencia de «feedback».

De cualquier modo, todo tipo de evaluación funcional, las innumerables medidas del lactato, los análisis de gran nivel, permitidas por las técnicas de videorregistración, son válidas solo parcialmente si, contemporáneamente, no se añaden análisis de las causas a través de las cuales se trata de individualizar cuales han sido las condiciones que han permitido que fuesen realizadas las prestaciones que han sido evaluadas. De hecho, solo los datos de la evaluación funcional sumados a los datos del entrenamiento realizado permiten obtener motivos objetivos que llevan a las decisiones sobre el entrenamiento.

Este circuito regulador, necesario para tomar decisiones sobre el entrenamiento fundadas en datos objetivos, deberá realizarse a través de la segunda parte de la "cadena operativa»».

Esta segunda parte, según ellos, consiste en que la toma de decisiones sobre el entrenamiento se base no solamente en los datos de la evaluación funcional, sino también teniendo en cuenta la referencia $a$ las causas que han llevado al desarrollo de las prestaciones.

A esta segunda parte de la «cadena operativa» lo llaman sistema consultores-entrenador y lo definen así:

«el sistema «consultores-entrenador» es el punto de encuentro entre ciencia y práctica. Su acción se basa sobre informaciones específicas o bien individualizadas, con elevada exigencia de objetividad, obtenidas del análisis de las competiciones, del entrenamiento en centros dotados de instrumentos de medida y de análisis del entrenamiento. Tales informaciones, filtradas de modo que sean presentadas de forma comprensible, teniendo en cuenta quién debe utilizarlas (atleta, entrenador), deben tener un contenido aplicable, relevante desde el punto de vista práctico y deben estar disponibles oportunamente para las decisiones sobre el entrenamiento y sobre la competición que debe tomar el entrenador.

En la implantación de este sistema tiene una importancia determinante la necesidad de información manifestada por la comunidad de los entrenadores».

i..... Y en estas estamos!

\section{¿Hay algo mas?}

«iEl hombre! El hombre, el principal factor de determinación de la actividad. Constituye un valor esencial y servirlo exige tomar iniciativas siempre nuevas.»

(Juan Pablo II) 
En el siglo XIX, un famoso fisiólogo español, LETAMENDI, decía: «A la tal fisiología humana, fáltale hombre, sóbrale rana». No es que a la ciencia del entrenamiento le falte hombre, pero tengo que decir que sin el hombre, no es casi nada.

Un error frecuente de los entrenadores es que confiamos mas en nuestros métodos que en el hombre. No se puede matematizar el entrenamiento, este se basa en principios científicos, pero es un «arte», (menor, ipor supuesto!), y debe ser modificado constantemente según necesidades del hombre al cual queremos entrenar.

Existe un «emocionario» del entrenamiento. El «secreto» del entrenador y de su éxito está en encontrar personas que quieran conseguir algo. Los resultados deportivos depende sobre todo de tener un ideal. No existe el «atleta de diseño». La clave de todo es el deportista, y «su» participación en el entrenamiento.

La individualización de la carga de entrenamiento ha sido siempre una de las mayores obsesiones de los investigadores de la teoría y práctica del entrenamiento. Pues bien, pese a todas los logros en este elemento, básico de la programación del entrenamiento, en mi opinión, la verdadera individualización solo el atleta la puede aportar. No se trata de volúmenes o intensidades, es la participación intelectual y volitiva del atleta, el «contenido emocional» de lo que hace, quien determina la calidad del estímulo de entrenamiento y su profundidad.

Él y solo él determina los limites humanos, ique tantas veces han tenido que ampliar los fisiólogos! Los límites humanos dependen de la voluntad del atleta, y según he leído al admirado CANDIDO, «la voluntad es la única potencia que el ser humano se puede dar a sí mismo».

Mi experiencia me ha hecho constatar que los grandes atletas tienen todos la misma máxima: « ¡COMO NO SABIAN QUE ERA IMPOSIBLE, LO HICIERON!» ... luego viene la ciencia y ... corrobora que se puede hacer.

Por último, unas palabras sobre el entrenador. Creo que es mas un artesano que un científico. Los métodos de entrenamiento no tienen la exactitud de las ciencias matemáticas. Una buena parte de lo que los técnicos hacen es discutible. Se puede decir que en su trabajo los técnicos deportivos deben proceder, necesariamente, por errores y aproximaciones. Siguen siendo intuitivos y empíricos. Maurice Genevoix en su novela "Vaincre à Olympie», describe así al «alipte» (equivalente griego del entrenador de atletismo actual) Antaeos, que hacia un viaje a través de Grecia buscando nuevos talentos, y que observaba a Euthymos, futuro campeón olímpico: «... una mirada más aguda que la flecha del arco. Hombre piadoso, artista, siempre tendiendo hacía lo divino, 


\section{Bases científicas del entrenamiento deportivo}

había reconocido en este hombre (Euthymos) el signo misterioso del héroe. Antaeos, peregrino por la fuerza de la edad, infatigable, paseaba su alforja a través de Grecia y las islas, en búsqueda de adolescentes nacidos bajo una estrella afortunada y que el favor de los dioses predestinase a la gloria olímpica».

.... Y poco o nada ha cambiado. El alipte-entrenador hoy, sigue siendo básicamente un educador, pero jay! ... hoy lo educativo no se lleva, no tiene valor en la actual sociedad. ¡Olvidaba señalar el cambio más significativo, la alforja que hoy paseamos infatigablemente, es de plástico y lleva la inscripción ¡Melbourne 56!.

\section{GRÁFICO 1}

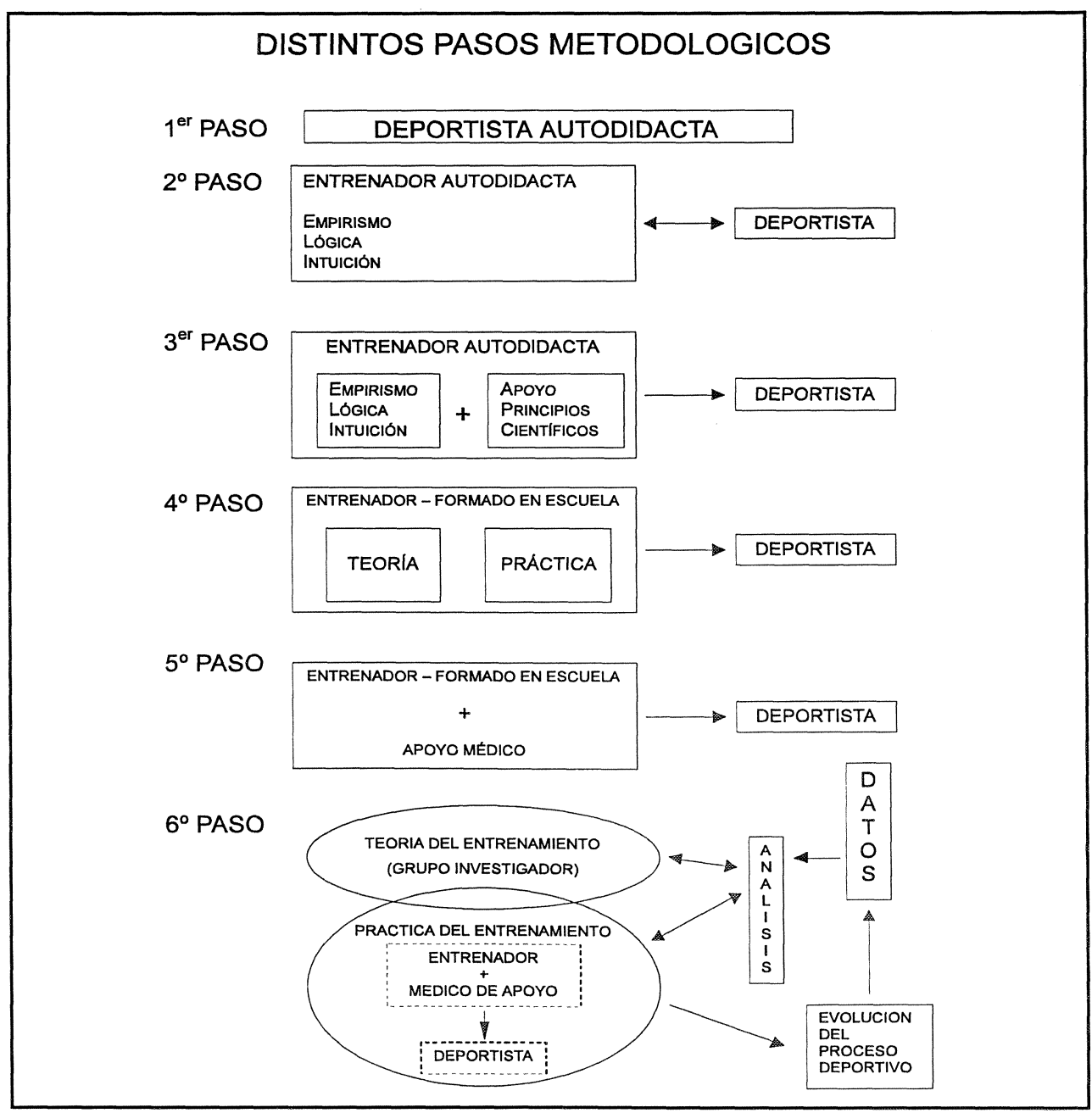




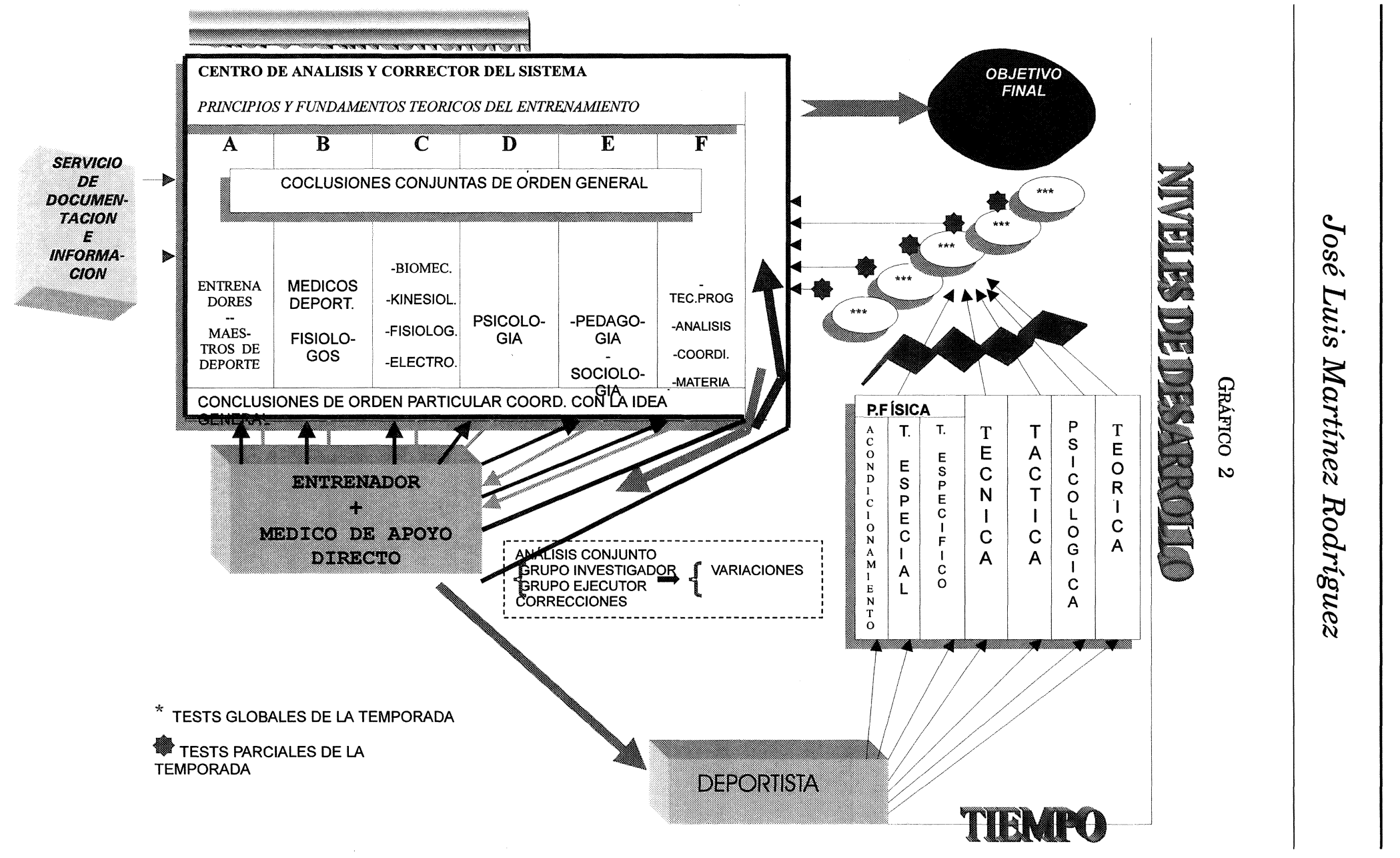




\section{Bases científicas del entrenamiento deportivo}

\section{Bibliografía}

Berger, Marcel et Moussat Emile: anthologie des textes sportifsdel'antiquite. Grasset B. Paris 1927.

Clare, Michael: introduction ou sport- Collection. "Vivre son temps». Les Editions Ouvrieres. Paris 1965

DiEm, Carl: historia de los deportes. Luis de Caralt. Editor. Barcelona 1966.

Genevorx, Maurice: Vaincra olympie. Union Generale d'Editions. Paris 1969.

Gillet, Bernard. «que saise?»: Historie du sport. Press Universitaires de France. Paris 1960.

Harris, Marvin: Nuestra especie. Alianza Editorial. Madrid 1995.

Lain Entralgo, P.: El cuerpo humano. Espasa. Universidad. Madrid 1989.

Lucot, Hubert: Le sport faut-il des surhommes? La Nouvelle Encyclopedie. Librairie Hachette, 1967.

Madariaga, Salvador: La ciencia. Los domingos de ABC. 1 de diciembre 1974.

MARTIN, Dietrich; KRUG, Jungen; ReIB, Manfred; Rost, Klaus: La evolución del sistema de entrenamiento y de competición en el deporte de élite y consecuencias para el ciclo olímpico, 1996-2000. Leistungssport, 1-1997, 25-31.

Montanelli, Indro: Historia de los griegos. Plaza y Janes, Editores. Barcelona 1963.

Ortega y Gasset: El origen deportivo del Estado. Revista Europea.

PaC-Pomarnacky: El atleta, un ser humano. "Sport Wyczyvowy", 1996.

Tschiene, Peter: La teoría dell'allenamento con o senza una priorita? SDS. Rivista di Cultura Sportiva, Anno XI, número 25.

ZANON, Sergio: Fondamenti di una nuova teoria dell'allanamento per lo sport competitivo. Atleticastudi. JulioAgosto 1992. 